\title{
EPIMORPHISMS OF COMPACT GROUPS ARE ONTO
}

\section{DETLEV POGUNTKE}

\begin{abstract}
ABSTRACr. It is proved that the epimorphisms in the category of compact groups are surjective. The proof is based on the representation theory of compact groups, especially on the well-known fact, that for a closed proper subgroup $H$ of a compact group $G$ there exists an irreducible representation of $G$ which, when restricted to $H$, contains the unit representation.
\end{abstract}

In any category a morphism $e: X \rightarrow Y$ is an epimorphism if $f e=g e$ $(f, g$ any morphisms starting at $Y$ ) implies $f=g$. In this connection the problem arises of characterizing epimorphisms in specific categories. In many concrete categories the epimorphisms are surjective, for instance in the category of groups, of finite groups, of modules over a ring and of topological spaces, but there are also concrete categories with nonsurjective epimorphisms, e.g. the category of rings or of Hausdorff topological spaces. In this note we will show that the epimorphisms of compact groups are surjective.

I wish to express my thanks to Professor K. H. Hofmann for his suggestion to characterize the epimorphisms in the category of compact groups.

Theorem. Let $G$ and $H$ be compact groups. A continuous homomorphism $f: H \rightarrow G$ is an epimorphism in the category of compact groups and continuous homomorphisms iff $f$ is surjective.

Since the proof of the theorem is based on the representation theory of compact groups, we recall the definition of a $G$-module in order to introduce the terminology used in this paper.

Definition. Let $G$ be a compact group. A $G$-module is a pair $(V, h)$ consisting of a finite-dimensional complex Hilbert space $V$ and a continuous homomorphism $h: G \rightarrow U(V)$ where $U(V)$ denotes the group of unitary transformations of $V$.

PROOF OF THE THEOREM. The "if"-part of the theorem is trivial. Suppose now, that $f$ is an epimorphism. Then the inclusion map from the compact group $f(H)$ into $G$ is an epimorphism, too. It is, therefore, no loss of generality to assume that $H \subseteq G$ and $f$ is the inclusion map. We must show $H=G$.

Received by the editors February 20, 1970.

AMS 1968 subject classifications. Primary 2260, 2210.

Key words and phrases. Epimorphisms, category, compact groups, finite-dimensional representations, unitary transformations. 
Lemma. If $(V, h)$ is a G-module and $W$ is an $H$-submodule of $(V, h)$ (i.e. a vector subspace of $V$ such that $h(H)(W) \subseteq W$ ), then $W$ is a $G$ submodule of $(V, h)$.

Proof of the Lemma. Let $M$ be the orthogonal complement of $W$ in $V$. Then $M$ is a $H$-submodule of $(V, h)$. Denote by $d$ the unitary (as it is easily verified) transformation $\left(1_{W},-1_{M}\right): W \oplus M \rightarrow W \oplus M$ and by $I_{d}$ the inner automorphism on $U(V)$ induced by $d$. Simple computations show (i) $h \cdot f=I_{d} \cdot h \cdot f$. Since $U(V)$ is compact and $f$ is an epimorphism in the category of compact groups we deduce from (i) that (ii) $h=I_{d} \cdot h$. In order to show that $W$ is a $G$-submodule of $(V, h)$ take any $g \in G$ and any $w \in W$ and write $h(g)(w)=\hat{w}+m$ with $\hat{w} \in W$ and $m \in M$. We must show that $h(g)(w) \in W$, or, equivalently, $m=0$. From (ii) and the fact that $W$ is the fixed point set of the involution $d$ it follows that $\hat{w}+m=h(g)(w)=\left(d \cdot h(g) \cdot d^{-1}\right)(w)=(d \cdot h(g))(w)$ $=d(\hat{w}+m)=\hat{w}-m$; hence $m=0$. From the Lemma we will obtain an indirect proof for $H=G$. Suppose, that $H$ is a proper subgroup of $G$. Then there exists a nontrivial irreducible $G$-module $(V, h)$ such that $W:=\{x \in V: h(g)(x)=x$ for each $g \in H\}$ is a nonzero $H$-submodule of $(V, h)$ (see $[1$, p. 28, Chapter 3] and note that every $G$-module is a direct sum of irreducible ones, or [2, Chapter VI, §VII, Proposition 5 ], for the Lie group case, the conditions of the latter proposition are satisfied by Theorem 4 of $\S$ XII in the same chapter of [2]). Denote by $c$ the constant homomorphism from $G$ in $U(V)$. From the Lemma and the irreducibility of $(V, h)$ it follows that $V=W$, and consequently $h \cdot f=c \cdot f$. Since $f$ is an epikorphism in the category of compact groups and $h$ and $c$ are in this category, we conclude $h=c$, in contradiction to the nontriviality of $(V, h)$. This contradiction completes the proof of the theorem.

\section{REFERENCES}

1. L. Auslander, Unitary representations of locally compact groups, Lecture Note, Yale University, New Haven, Conn., 1961/62.

2. C. Chevalley, Theory of Lie groups, Princeton Math. Series, vol. 8, Princeton Univ. Press, Princeton, N. J., 1946. MR 7, 412.

Universität Bielefeld, 48 Bielefeld, West Germany 